\title{
Quantitative depth-resolved photoelectron spectroscopy analysis of the interaction of energetic oxygen ions with the beryllium-tungsten alloy $\mathrm{Be}_{2} \mathrm{~W}$
}

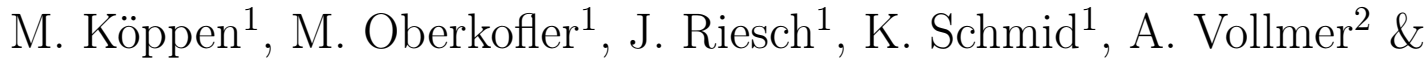 \\ Ch. Linsmeier*1 \\ ${ }^{1}$ Max-Planck-Institut für Plasmaphysik, EURATOM Association, Boltzmannstr. 2, 85748 Garching b. \\ München, Germany \\ ${ }^{2}$ Helmholtz-Zentrum Berlin für Materialien und Energie GmbH, Wilhelm-Conrad-Röntgen-Campus BESSY II, \\ Albert-Einstein-Str. 15, 12489 Berlin, Germany
}

November 14, 2012

\begin{abstract}
The chemical reactions during the implantation of oxygen ions into a thin berylliumtungsten alloy layer on a beryllium substrate and subsequent annealing are investigated by depth-resolved chemical analysis using X-ray photoelectron spectroscopy. By using variable photon energies at a synchrotron, identical kinetic energies of the photoelectrons from the different core levels are selected. This enables a chemical analysis at identical depth intervals for all core levels. From these sets of photoelectron spectra chemically resolved depth profiles are determined. The depth profiles allow an interpretation of the reaction and diffusion steps during implantation and annealing steps. This leads to a detailed understanding of the complex processes in a multi-component solid.
\end{abstract}

\section{Introduction}

The first wall of current and in particular future fusion devices applies different elements at the plasma-facing components. For ITER, currently beryllium and tungsten are the materials of choice for first wall and divertor, respectively. In addition, carbon might be used at the divertor strike points [1]. In addition to these elements from the wall components, impurities from the plasma, either intentionally added like seeding gases $\left(\mathrm{Ar}, \mathrm{N}_{2}\right)$ or impurities like oxygen, can contribute to the formation of compounds or alloys during operation (formation of 'mixed materials'). During plasma operation, wall materials are eroded, transported in the plasma, and re-deposited in different areas of the plasma vessel. Elevated temperatures and the kinetic

*corresponding author's e-mail address: linsmeier@ipp.mpg.de 
energy of the deposited particles can trigger the formation of these mixed materials. Since in ITER the major part of the first wall will consist of beryllium, its deposition on the tungsten surfaces in the baffle and divertor regions is a probable process during operation. The two metals can alloy due to elevated temperatures in these areas and form $\mathrm{Be}_{2} \mathrm{~W}[2]$. Together with oxygen as a plasma impurity, the formation of the respective oxides can be triggered both by thermally and kinetically induced chemical reactions. These chemical phases show strongly altered properties compared to the original wall materials. For beryllium, the melting point of the oxide is $2780 \mathrm{~K}$ - significantly higher than that of the metal with $1551 \mathrm{~K}$. For tungsten, the formed oxides reduce the melting point from $3683 \mathrm{~K}$ for the metal to $1746 \mathrm{~K}$ and $1773 \mathrm{~K}$, depending on the formed oxides $\left(\mathrm{WO}_{3}\right.$ and $\mathrm{WO}_{2}$, respectively). The knowledge of the chemical processes at the first wall of a fusion device is therefore crucial for predicting operational scenarios. In previous studies, various binary systems of the first wall elements Be, $\mathrm{C}$, and $\mathrm{W}$ have been investigated [3]. Based on this knowledge, the mixed material formation processes taking into account plasma impurities are studied: In this work we investigate the chemical phases during oxygen implantation and annealing. Since new components strongly increase the complexity of the reactive system, new analysis techniques like a depth-resolved chemical analysis is required to analyze not only surface processes, but allow an extended characterization of reactive and diffusive processes. In an earlier paper we already showed that X-ray photoelectron spectroscopy using tuned photon energies from a synchrotron (ERXPS: energy-resolved X-ray photoelectron spectroscopy) allows a depth-resolved chemical analysis [4]. The tuned kinetic energy of the produced photons determines the their escape depth from the solid and therefore the information depth. We could show that the implantation of oxygen ions into a $\mathrm{Be}_{2} \mathrm{~W}$ alloy leads to the formation of different chemical compounds in various depths within the implantation range of the ions. In this paper, we extend the previous investigation towards a quantitative analysis of the composition in different depths. This allows for the first time a quantitative, non-destructive depth-resolved chemical analysis. Such an analysis is the basis for a deeper understanding of the reactive and diffusive processes during mixed material reactions.

\section{Experiment}

The experiment is carried out at the synchrotron facility HZB-BESSY II at the end station SurICat. For sample preparation our preparation chamber LAICA is used which allows vapor deposition of several elements, ion implantation, sample annealing, and Auger electron spectroscopy (AES) analysis. Details of the sample preparation and experimental conditions are given in [4]. Here, we repeat only the implantation and annealing steps which are carried out in situ: (1) Cleaning of the polycrystalline Be sample by Ar sputtering and annealing, (2) Deposition of $4 \mathrm{~nm} \mathrm{~W}$ on Be from the vapor phase and annealing at $900 \mathrm{~K}$ for $60 \mathrm{~min}$ to form a $\mathrm{Be}_{2} \mathrm{~W}$ alloy layer, (3) Oxygen implantation at $1000 \mathrm{~V}$ with a fluence of $1.49 \cdot 10^{16} \mathrm{~cm}^{-2}$, (4) Annealing at $600 \mathrm{~K}$ for $30 \mathrm{~min}$, (5) Oxygen implantation at $500 \mathrm{~V}$ with a fluence of $1.41 \cdot 10^{16} \mathrm{~cm}^{-2}$, (6) Annealing at $600 \mathrm{~K}$ for $30 \mathrm{~min}$, (7) Oxygen implantation at $500 \mathrm{~V}$ with a fluence of $1.29 \cdot 10^{16} \mathrm{~cm}^{-2}$. After each reaction step photoelectron spectra are measured in high resolution mode with photon energies providing six identical photoelectron kinetic energies for each core level under investigation ( $\mathrm{Be} 1 s, \mathrm{~W} 4 f$, and $\mathrm{O} 1 s$ ). The chemically resolved quantitative depth profiles determined from the spectra after steps $2-5$ and 7 are discussed in detail below. The oxygen implantation profiles are calculated with SDTrim.SP [5, 6], assuming a beam composition of 
$82 \% \mathrm{O}_{2}^{+}$and $18 \% \mathrm{O}^{+}[7]$. The implantation profiles are qualitatively identical to the profiles shown in [4]. The maximum $\mathrm{O}$ fractions for a total fluence of $1 \cdot 10^{16} \mathrm{~cm}^{-2}$ reach approximately 0.3 in case of the $500 \mathrm{~V}$ implantation and 0.24 for the $1000 \mathrm{~V}$ implantation.

In energy-resolved XPS the information depth is varied by using different photon energies to excite the photoelectrons from core levels. According to $E_{k i n}=h \nu-E_{b}-\phi_{a}$, a higher photon energy $h \nu$ results in a higher kinetic energy of the photoelectron $E_{k i n}$ for a given binding energy $E_{b}$. The work function of the analyzer $\phi_{a}$ is determined for each spectrum by a Au $4 f_{7 / 2}$ reference measurement. Since the inelastic mean free path of electrons is energy dependent, it is possible to change the information depth by using different photon energies [8]. In order to measure at identical information depths for all core levels of interest, the photon energy is tuned such that the resulting kinetic energy of photoelectrons for the $\mathrm{W} 4 f$, Be $1 s$, and $\mathrm{O} 1 s$ core levels is identical. The actually applied values are given in [4]. Binding energy (BE) scales of the spectra are referenced to the Au $4 f_{7 / 2}$ peak with a binding energy of $84.0 \mathrm{eV}$. For the $1 s$ core levels, the background in the XP spectra is subtracted by applying a Shirley-type background [9] and the peaks are fitted with pseudo-Voigt functions. The W $4 f_{7 / 2}$ peaks are fitted using a Doniach-Šnjić-style function [10] and a linear background. During the fits, the doublet separation between the $\mathrm{W} 4 f_{7 / 2}$ and $\mathrm{W} 4 f_{5 / 2}$ peaks is held constant at $2.18 \mathrm{eV}$, as is their area ratio of 1.33 .

The intensity measured for a specific core level is described by the following equation:

$$
I=\sigma \cdot L \cdot J \cdot D \cdot T \cdot \int_{z=0}^{\infty} \rho(z) \exp \left[-\frac{z}{\lambda \cos \alpha}\right]
$$

It contains a number of physical parameters describing the photon flux $J$, the asymmetry parameter $L$ and the photoionization cross sections $\sigma$ after Yeh [11], the analyzer transmission $T$ and the detector efficiency $D$ given by the spectrometer manufacturer. The contribution of the atoms in a specific chemical state is described by the integral over the depth-dependent particle density $\rho(z)$, exponentially weighted by the depth $z$, the inelastic electron mean free path $\lambda$ (IMFP) and the angle $\alpha$ between the surface normal and the analyzer $\left(\alpha=0^{\circ}\right.$ in our experiment). The exponential decay of the photoelectron intensity with depth means that the closer an atom is located to the surface, the higher is its contribution to the measured intensity I. Since the depth distribution of the elements in the sample is also effecting the IMFP, the determination of the depth profiles is a non-linear problem and can only be solved numerically. The IMFPs required for these calculations are determined after the model found by Gries [13]. This model allows to determine $\lambda$ in a consistent way not only for pure elemental and compound solids, but for arbitrary compositions. The composition determined by the XPS measurements is iteratively considered for the determination of the $\lambda$ used in the calculations of the ERXPS intensities.

A newly developed program based on equation (1) and the described parameters is used to determine ERXPS intensities by forward calculations for a given composition. Fitting the results of these forward calculations to the experimentally obtained normalized intensities and adapting the composition (and respective $\lambda$ values) creates depth profiles on a nanometer scale. This program is implemented in Mathematica which provides suitable numerical solver routines. A simulated annealing algorithm is used for the fit procedure. The experimental input are the peak areas determined from the measured spectra by peak fitting, as described above. After normalization with the appropriate parameters, these exponentially weighted areal densities are compared to the results from forward calculations by the Mathematica model which optimizes the elements in the calculated depth profile to fit the experimental results. For a valid depth 
profile the elements and compounds present in a numerical solution must reproduce the total volume of the entire depth profile within $5 \%$. This ensures that the calculated elemental depth distribution is a valid solution in terms of the detected chemical species. A detailed description of the model will be published separately.

\section{Results and Discussion}

From the peak fitting procedure, the chemical composition and the respective concentrations for the chemical phases (e.g. $\mathrm{Be}_{2} \mathrm{~W}, \mathrm{WO}_{3}, \mathrm{WO}_{2}$, or $\mathrm{W}$ ) are determined. Examples for fits are given in Fig. 1 for the $\mathrm{Be} 1 s$ and $\mathrm{W} 4 f$ core levels. In our earlier publication on the qualitative chemical analysis of these experiments [4], we assigned ternary Be-W-O compounds to some peaks. Given the discussion in that paper, this assignment is still a feasible solution and supported by the results from the measurements. In this quantitative analysis, however, we restrict the assignment of the peaks to elemental species and binary compounds only. This has two reasons: First, as extensively discussed in [4], the ternary Be-W-O compounds are deduced from analogous compounds and no direct reference measurements are available. Their existence is plausible from a qualitative analysis, as performed in [4]. Respective peak shifts exist both in the Be $1 s$ and $\mathrm{W} 4 f$ regions. However, since we now extend the analysis to a quantitative depth profile, the agreements with the respective peak integrals and in particular with the stoichiometries is less convincing for ternary compounds. Second, for the numerical fit procedure we limit the number of species as far as possible, and no values for the densities of the ternary compounds are available.

For the elements and binary compounds marked within Fig. 1, references values are available in the literature which support the assignments. In the Be $1 s$ region, three peaks are identified: $\mathrm{BeO}$ at a $\mathrm{BE}$ of $113.7 \mathrm{eV}, \mathrm{Be}_{2} \mathrm{~W}$ at $111.1 \mathrm{eV}$, and a third peak at $112.4 \mathrm{eV}$. This last signal is often observed on $\mathrm{Be}$ if $\mathrm{BeO}$ and a metallic species $\left(\mathrm{Be}\right.$ or $\left.\mathrm{Be}_{2} \mathrm{~W}\right)$ are present. Its interpretation is not unique, we assume that $\mathrm{BeO}$ in direct vicinity of metallic species leads to this shift. In the quantitative analysis, this intensity is therefore added to the $\mathrm{BeO}$ intensity (typically, the total $\mathrm{BeO}$ intensity is changed only by less than $10 \%$ by this peak at $112.4 \mathrm{eV})$. In the $\mathrm{W}$ ff region, five doublets are identified by the peak fitting routine. In Fig. 1b, the respective $W f_{7 / 2}$ peaks are plotted as solid lines, whereas the corresponding $\mathrm{W} 4 f_{5 / 2}$ peaks are plotted as dashed lines. At lowest $\mathrm{BE}$, the peak corresponding to $\mathrm{Be}_{2} \mathrm{~W}$ is located at a $\mathrm{BE}$ of $31.0 \mathrm{eV}$. At $31.4 \mathrm{eV}$ the peak of metallic $\mathrm{W}$ is observed. Tungsten in $\mathrm{WO}_{2}$ leads to the peak at $32.0 \mathrm{eV}$. The two peaks with highest BEs are both assigned to tungsten(VI)oxides and are treated in the quantitative analysis as one species. The peak at $35.3 \mathrm{eV}$ is assigned to $\mathrm{WO}_{3-\mathrm{x}}$ and the peak at $36.4 \mathrm{eV}$ to the stoichiometric oxide $\mathrm{WO}_{3}$. During the fitting procedure for all spectra of the whole experimental series, the peak positions are allowed to vary up to $0.9 \mathrm{eV}$ for the oxidic species and up to $0.1 \mathrm{eV}$ for the metallic species. This is consistent with the general observation in XPS that peaks originating from oxides are less exact in their absolute position than peaks originating from metals.

In the following paragraphs, the obtained depth profiles and the chemical reactions and diffusive processes taking place at the single experimental steps are described, beginning from the alloy. The resolution used for the depth profiles is three slices with a thickness of $1.4 \mathrm{~nm}$ each, resulting in a analyzed depth of $4.2 \mathrm{~nm}$. This means slice 1 corresponds $0-1.4 \mathrm{~nm}$ on the depth scale, slice 2 to $1.4-2.8 \mathrm{~nm}$ and slice 3 to $2.8-4.2 \mathrm{~nm}$. Within each slice, a homogeneous composition is assumed. 
For the alloyed sample, the obtained depth profile is shown in Fig. 2. In slices 2 and 3 only the alloy $\mathrm{Be}_{2} \mathrm{~W}$ is visible. At the surface of the sample $\mathrm{BeO}$ is found. Since the precursor for the alloy is a Be disc covered by a $\mathrm{W}$ layer, Be must diffuse during the alloy formation through the tungsten or the newly formed alloy layer to the surface, where it gets oxidized by residual gas even under the good vacuum conditions in the preparation chamber.

In the first implantation step oxygen is implanted with an acceleration voltage of $1000 \mathrm{~V}$ and a fluence of $1.49 \cdot 10^{16} \mathrm{~cm}^{-2}$ in $\mathrm{Be}_{2} \mathrm{~W}$. The determined depth profile is shown in Fig. 3. The decomposition of the alloy is observed in the sample except for a remaining volume fraction of $46 \%$ in slice 2. Be from the decomposed alloy is oxidized completely. The released tungsten from the alloy is converted to its two oxidic states $\mathrm{WO}_{2}$ and $\mathrm{WO}_{3}$, as well as to its metallic form. The Be-containing compounds are located in the upper two slices, while tungsten and its oxides can be found in the lowest slice. This points to the diffusion of metallic Be towards the surface.

After the sample is annealed for $30 \mathrm{~min}$ at $600 \mathrm{~K}$, a reduction of tungsten oxides is observed. The depth profile is shown in Fig. 4. $\mathrm{No}_{\mathrm{WO}_{3}}$ is visible anymore and only $\mathrm{WO}_{2}$ is detected in slice 2. The amount of tungsten bound in the $\mathrm{Be}_{2} \mathrm{~W}$ alloy increases, slice 2 consists entirely of it. The only reducing agent available in the sample is metallic Be. Additional metallic beryllium can only be found in the bulk beyond the information depth of the experiment. Therefore Be must diffuse from the bulk towards the surface, where it can react with the tungsten oxides to form $\mathrm{BeO}$ and providing $\mathrm{W}$ for the alloy formation. The reduced tungsten forms again the alloy $\mathrm{Be}_{2} \mathrm{~W}$ with $\mathrm{Be}$.

The next sample treatment within the experiment is the implantation of $\mathrm{O}$ ions at an acceleration voltage of $500 \mathrm{~V}$ and an $\mathrm{O}$ fluence of $1.41 \cdot 10^{16} \mathrm{~cm}^{-2}$. The total $\mathrm{O}$ fluence from all implantations sums up to $2.90 \cdot 10^{16} \mathrm{~cm}^{-2}$. In the chemically resolved depth profile after this implantation step (Fig. 5) the decomposition of $\mathrm{Be}_{2} \mathrm{~W}$ is visible, compared to the previous sample composition (Fig. 4). Only small remains of the alloy are visible in slices 2 (4 vol.- $\%)$ and $3(24$ vol.- $\%)$. The released Be is completely oxidized to $\mathrm{BeO} . \mathrm{W}$ from $\mathrm{Be}_{2} \mathrm{~W}$ is mostly oxidized to $\mathrm{WO}_{2}$, which is with 49 vol.-\% predominantly present in slice 3. Small amounts of W (9 vol.- $\%$ in slice 2, 4 vol.- $\%$ in slice 3 ) are present in the metallic state.

After the first implantation step with $500 \mathrm{eV}$ the sample is annealed for 30 min at $600 \mathrm{~K}$. Following this temperature step, $\mathrm{O}$ ions are additionally implanted with an acceleration voltage of $500 \mathrm{~V}$ and a fluence of $1.29 \cdot 10^{16} \mathrm{~cm}^{-2}$. At this step, the sample is now implanted with a total O fluence of $4.19 \cdot 10^{16} \mathrm{~cm}^{-2}$ and the chemically resolved depth profile is shown in Fig. 6. In comparison to the depth profile of the previous step (Fig. 5), a lower volume ratio of tungstencontaining compounds is detected in the slices 2 and 3: In slice 2, no $\mathrm{W}$ species are detected and in slice $3 \mathrm{Be}_{2} \mathrm{~W}, \mathrm{~W}$ and $\mathrm{WO}_{2}$ sum up to 53 vol.- $\%$. Therefore, Be has to diffuse to the surface where it is oxidized. In slice 3 remains of $\mathrm{Be}_{2} \mathrm{~W}(21$ vol.- $\%)$ and $\mathrm{W}$ (19 vol.- $\left.\%\right)$ are detected. Except for these metallic phases the analyzed depth of the sample consists entirely of oxidic species.

Some basic reaction and diffusion patterns can be derived from the evaluation of the obtained depth profiles. Be diffuses always in the direction of available oxygen to form $\mathrm{BeO}$. If no oxygen is provided by implantation, the Be diffuses to the top of the sample to react with (limited amounts of) oxidic species in the residual gas. W does not act as a diffusion barrier. Oxygen implantation leads to the decomposition of the alloy and to immediate formation of $\mathrm{BeO}$. The released tungsten from the decomposed alloy forms oxides if additional unbound oxygen is available. The formation of $\mathrm{WO}_{2}$ is observed when the available oxygen amount is insufficient to form $\mathrm{WO}_{3}$. Oxygen preferentially reacts with $\mathrm{Be}$ to $\mathrm{BeO}$ rather than forming tungsten 
oxides. This seems to contradict with the standard enthalpies of formation $\Delta_{f} H_{\text {solid }}^{\circ}$ for $\mathrm{BeO}$ of $-609 \mathrm{~kJ} \mathrm{~mol}^{-1}$ and for $\mathrm{WO}_{3}$ of $-843 \mathrm{~kJ} \mathrm{~mol}^{-1}$ (data taken from [14]), since $\mathrm{WO}_{3}$ is the most exothermic compound. However, when normalizing these enthalpy values to a formula unit $\mathrm{O}_{2}$, it becomes obvious that $\mathrm{BeO}$ is the most exothermic compound. For $\mathrm{BeO}$, the energy gained per $\mathrm{O}_{2}$ is $1218 \mathrm{~kJ} \mathrm{~mol}^{-1}$, while for $\mathrm{WO}_{3}$ it is only $562 \mathrm{~kJ} \mathrm{~mol}^{-1}$. Consequently, Be readily reacts with available oxygen and no metallic Be is detected in the depth profiles after $\mathrm{O}$ implantations. Annealing after the first implantation results in the re-formation of $\mathrm{Be}_{2} \mathrm{~W}$ alloy. A reduction of the perviously present $\mathrm{W}$ oxides by Be from the substrate, acting as a Be reservoir, is plausible.

\section{Conclusions}

The chemical reactions during the implantation of oxygen into a $\mathrm{Be}_{2} \mathrm{~W}$ alloy layer on a beryllium substrate are analyzed by energy-resolved XPS in a quantitative way. During the oxygen implantation, $\mathrm{BeO}$ and tungsten oxides formed. After annealing, $\mathrm{BeO}$ is the dominating oxide as the thermodynamically most stable compound. Tungsten reacts again with Be from the substrate to form $\mathrm{Be}_{2} \mathrm{~W}$. A numerical analysis of the chemical species determined by peak fitting allows the calculation of a depth profile of all species present within the information depth of XPS. This allows for the first time to observe reaction and diffusion processes in a multi-component solid in a non-destructive way.

\section{Acknowledgements}

We are grateful to T.-V. Phan and H. Löchel for their support during the beam time at HZBBESSY II. This work has partially been funded in the frame of the FEMaS project within the European Community's Seventh Framework Programme (FP7 / 2007-2011) under Grant Agreement 224752.

\section{References}

[1] G. Federici, A. Loarte, G. Strohmayer, Plasma Phys. Control. Fusion. 2003, 45 (9), 15231547

[2] A. Wiltner and Ch. Linsmeier, New J. Phys. 2006, 8, 181

[3] Ch. Linsmeier, K. Ertl, J. Roth, A. Wiltner, K. Schmid, F. Kost, S. R. Bhattacharyya, M. Baldwin, R. P. Doerner, J. Nucl. Mater. 2007, 363-365, 1129-1137

[4] M. Köppen, J. Riesch, A. Vollmer, Ch. Linsmeier, Phys. Scr. 2011, T145, 014015

[5] W. Eckstein, R. Dohmen, A. Mutzke, R. Schneider, SDTrimSP: A Monte-Carlo Code for Calculating Collision Phenomena in Randomized Targets, Max-Planck-Institut für Plasmaphysik, IPP-Report 12/3 2007

[6] W. Eckstein, Computer Simulation of Ion-Solid Interactions, Springer Series in Materials Science, Vol. 10, Springer, Berlin 1991 
[7] NIST Mass Spec Data Center, S.E. Stein (director), NIST Chemistry Webbook, NIST Standard Reference Database Number 69, Chapter Mass Spectra, National Institute of Standards and Technology, Gaithersburg MD 2011

[8] F. Kost, Ch. Linsmeier, M. Oberkofler, M. Reinelt, M. Balden, A. Herrmann, S. Lindig, J. Nucl. Mater. 2009, 390-391, 975-978

[9] D. A. Shirley, Phys. Rev. B. 1972, 5, 4709-4714

[10] S. Doniach, M. Šunjić, J. Phys. C: Solid State 1970, 3(2), 285-291

[11] J. J. Yeh, I. Lindau, At. Data Nucl. Data Tables 1985, 32(1), 1-155

[12] M.P. Seah, W.A. Dench, Surf. Interface Anal. 1979, 1, 2-11

[13] W. H. Gries, Surf. Interface Anal. 1996, 24, 38-50

[14] M. W. Chase, J. Phys. Chem. Ref. Data 1998, Monograph 9, 1-1951 

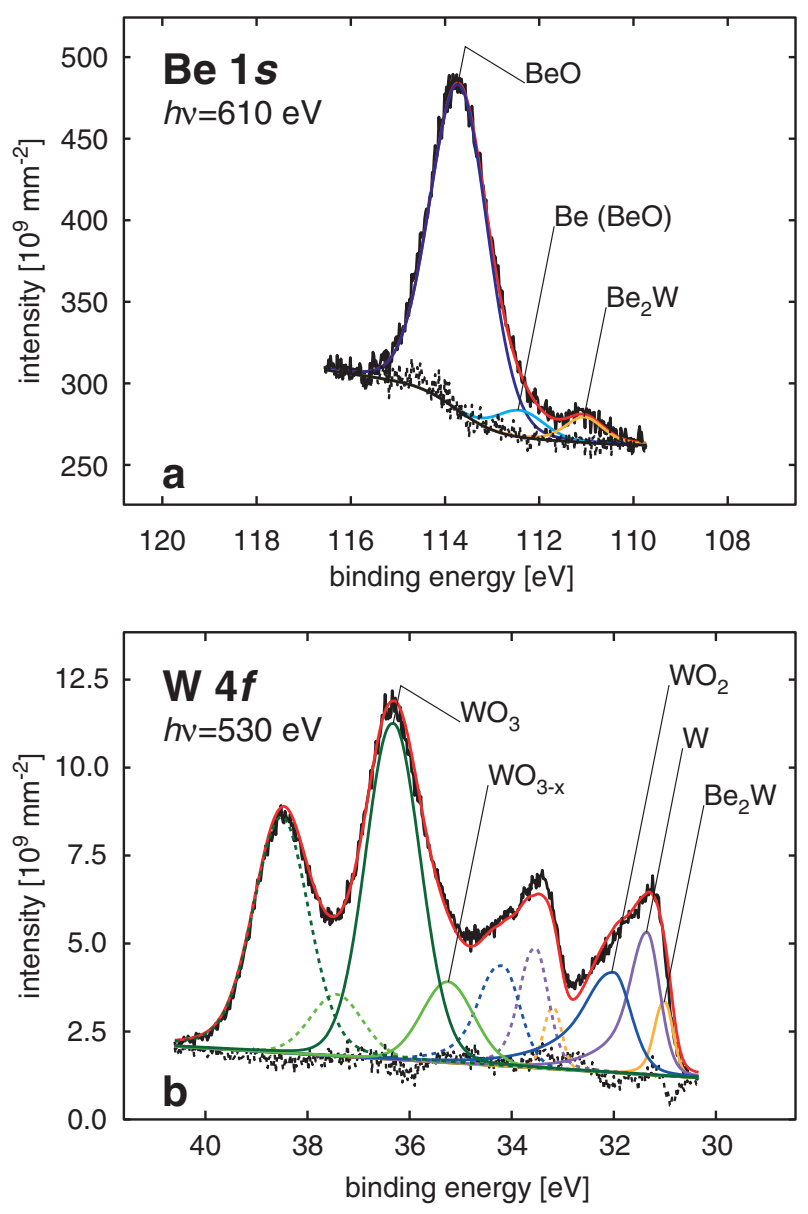

Figure 1: XPS spectra in the Be $1 s$ (a) and W $4 f(\mathrm{~b})$ core level regions after implanting oxygen at $1000 \mathrm{~V}$ and $500 \mathrm{~V}$ into $\mathrm{Be}_{2} \mathrm{~W}$ (preparation step 5, see text). The kinetic energy of the photoelectrons is approximately $490 \mathrm{eV}$ in both cases. The detected chemical species are labeled. Shown are the measured data, the single fit functions and their sum, together with the background and the difference between data and fit sum. 


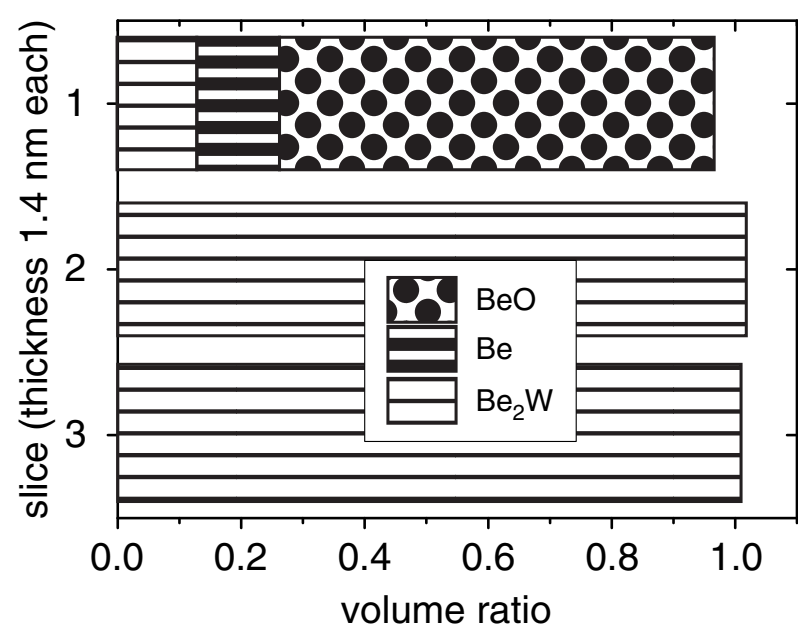

Figure 2: Chemically resolved depth profile of the $\mathrm{Be}_{2} \mathrm{~W}$ alloy layer on beryllium.

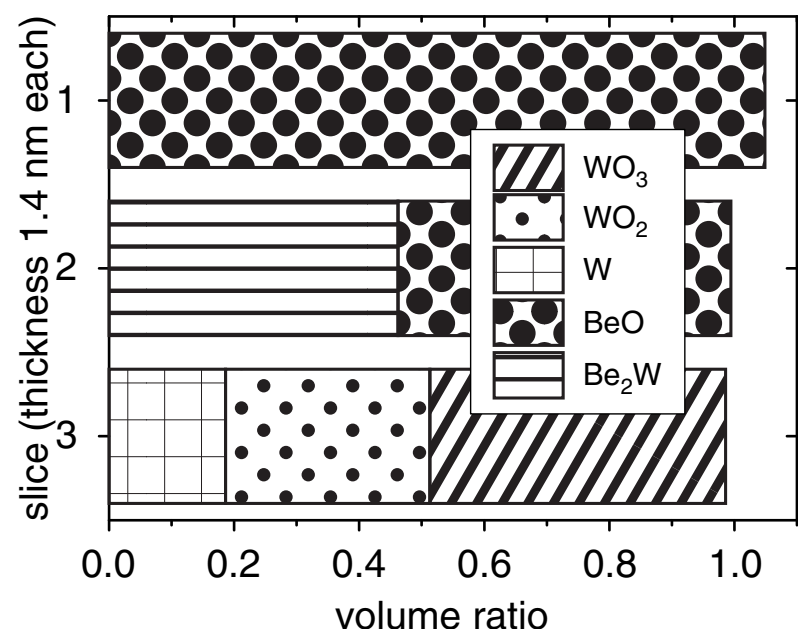

Figure 3: Chemically resolved depth profile after implantation of oxygen at $1000 \mathrm{~V}$ acceleration voltage at a fluence of $1.49 \cdot 10^{16} \mathrm{~cm}^{-2}$. 


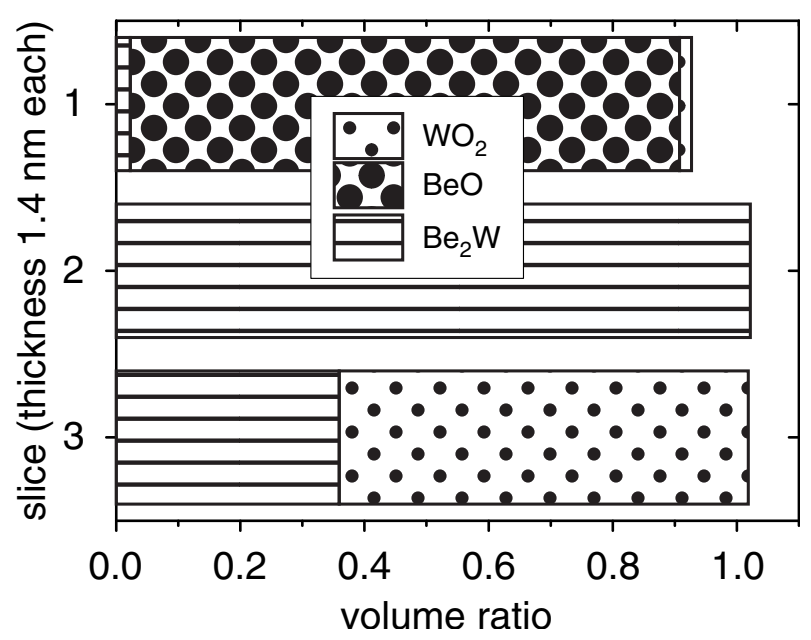

Figure 4: Chemically resolved depth profile after an additional annealing step at $600 \mathrm{~K}$ for 30 min.

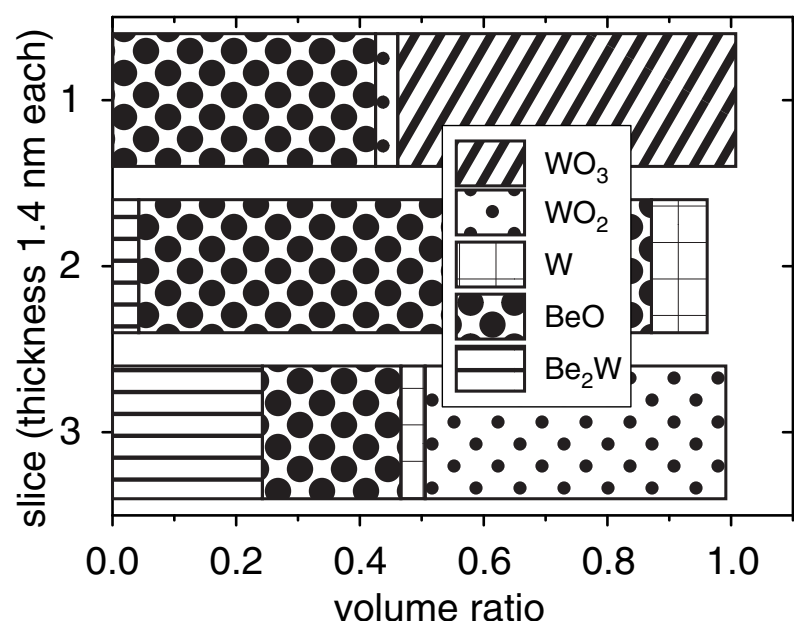

Figure 5: Chemically resolved depth profile sample after O implantation with $500 \mathrm{~V}$ acceleration voltage at a fluence $1.41 \cdot 10^{16} \mathrm{~cm}^{-2}$. The total $\mathrm{O}$ fluence is $2.90 \cdot 10^{16} \mathrm{~cm}^{-2}$. 


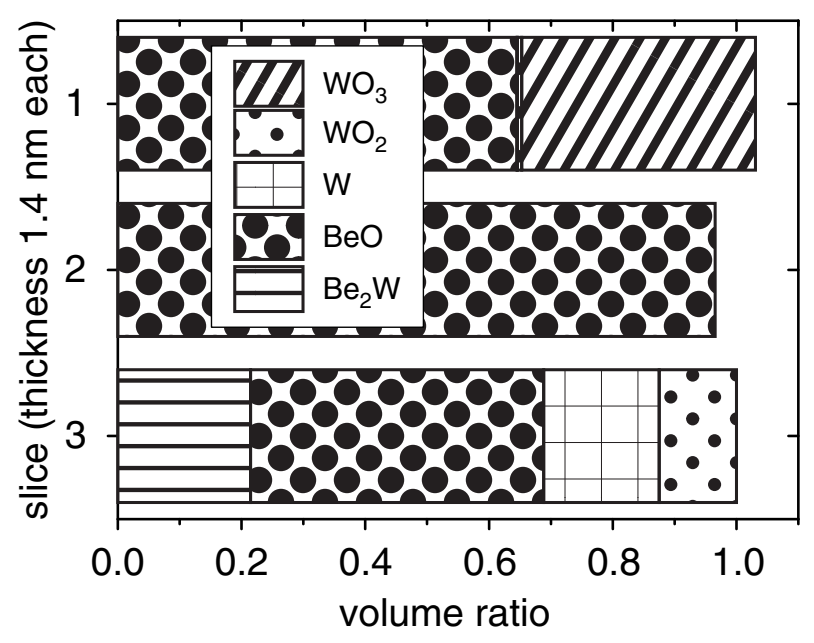

Figure 6: Chemically resolved depth profile after the second $\mathrm{O}$ implantation with $500 \mathrm{~V}$ acceleration voltage and a fluence $1.29 \cdot 10^{16} \mathrm{~cm}^{-2}$. The total $O$ fluence is $4.19 \cdot 10^{16} \mathrm{~cm}^{-2}$. 